\title{
Enhanced in vitro and in vivo therapeutic efficacy of codrug-loaded nanoparticles against liver cancer
}

This article was published in the following Dove Press journal:

International Journal of Nanomedicine

28 September 2012

Number of times this article has been viewed

\begin{abstract}
Xiaolin $\mathrm{Li}^{1}{ }^{1, *}$
Hua'e $\mathrm{Xu}^{2, *}$

Xinzheng $\mathrm{Dai}^{3,4, *}$

Zhenshu Zhu ${ }^{5}$

Baorui Liu ${ }^{6}$

Xiaowei Lu'

'Department of Geriatrics,

${ }^{2}$ Department of Pharmacy, the First Affiliated Hospital to Nanjing Medical University, Nanjing; ${ }^{3}$ Key Laboratory of Living Donor Liver Transplantation, Ministry of Public Health, ${ }^{4}$ Liver Transplantation Center, the First Affiliated Hospital to Nanjing Medical University, Nanjing; ${ }^{5}$ Department of Pharmaceutical Analysis, China Pharmaceutical University, Nanjing; ${ }^{6}$ The Comprehensive Cancer Center of Drum Tower Hospital, Medical School of Nanjing University and Clinical Cancer Institute of Nanjing University, Nanjing, China
\end{abstract}

*These authors contributed equally to this work

Correspondence: Baorui Liu

The Comprehensive Cancer Center of Drum Tower Hospital, Medical School of Nanjing University and Clinical Cancer Institute of Nanjing University, Nanjing 210008, China

Tel +86 258310708 I

Fax +86258310 708I

Email baoruiliu@nju.edu.cn

Xiaowei Lu

Department of Geriatrics, the First Affiliated Hospital of Nanjing Medical University, Nanjing 210029, China

$\mathrm{Tel}+862568135131$

$\mathrm{Fax}+862568135131$

Email njluxiaowei@।63.com

\begin{abstract}
Paclitaxel (Ptx), one of the most widely used anticancer agents, has demonstrated extraordinary activities against a variety of solid tumors. However, the therapeutic response of Ptx is often associated with severe side effects caused by its nonspecific cytotoxic effects and special solvents (Cremophor $\mathrm{EL}^{\circledR}$ ). The current study reports the stable controlled release of Ptx/tetrandrine (Tet)-coloaded nanoparticles by amphilic methoxy poly(ethylene glycol)-poly(caprolactone) block copolymers. There were three significant findings. Firstly, Tet could effectively stabilize Ptxloaded nanoparticles with the coencapsulation of Tet and Ptx. The influence of different Ptx/Tet feeding ratios on the size and loading efficiency of the nanoparticles was also explored. Secondly, the encapsulation of Tet and Ptx into nanoparticles retains the synergistic anticancer efficiency of Tet and Ptx against mice hepatoma H22 cells. Thirdly, in the in vivo evaluation, intratumoral administration was adopted to increase the site-specific delivery. Ptx/Tet nanoparticles, when delivered intratumorally, exhibited significantly improved antitumor efficacy; moreover, they substantially increased the overall survival in an established H22-transplanted mice model. Further investigation into the anticancer mechanisms of this nanodelivery system is under active consideration as a part of this ongoing research. The results suggest that Ptx/Tet-coloaded nanoparticles could be a potential useful chemotherapeutic formulation for liver cancer therapy.
\end{abstract}

Keywords: tetrandrine, paclitaxel, nanoparticle, liver cancer

\section{Introduction}

Liver cancer is one of the most frequently diagnosed cancers worldwide, with relatively high morbidity and mortality. ${ }^{1}$ Half of the newly diagnosed cases and deaths were estimated to occur in China. ${ }^{2}$ However, due to the resistance to conventional chemotherapy, the overall survival of patients with liver cancer remains poor.

Paclitaxel (Ptx), one of the most widely used anticancer agents, has demonstrated extraordinary activities against a variety of solid tumors. Ptx exerts its cytotoxicity by disrupting cell functions through accelerating the microtubule assembly from tubulin and blocking the depolymerization of the microtubule, which eventually causes a G2-M cell arrest. ${ }^{3}$ Recent clinical trials have demonstrated the advantage of Ptx in combination with other anticancer agents in treatment of hepatocellular carcinoma. ${ }^{4,5}$ However, the therapeutic response of Ptx is often associated with severe side effects caused by its nonspecific cytotoxic effects and special solvents $\left(\text { Cremophor } \mathrm{EL}^{\circledR}\right)^{6,7}$ Therefore, it becomes the focus in the field of cancer therapy to search novel strategies in order to achieve higher antitumor efficacy of Ptx.

Tetrandrine (Tet), a bis-benzylisoquinoline alkaloid isolated from the root of hang-fang-chi (Stephania tetrandra S Moore), is used in traditional Chinese medicine 
as an antirheumatic, antiinflammatory, and antihypertensive agent. ${ }^{8}$ Recent studies have shown that Tet exhibited antitumor effects in vitro and in vivo. ${ }^{9-11}$ Moreover, several studies reported the multidrug resistance reversal effect of Tet in cancer cells and animal models. ${ }^{12-14}$ In a previous report, it was demonstrated that Tet could synergistically increase the cytotoxicity of Ptx against gastric cancer cells. ${ }^{15}$ These findings demonstrated the potential of Tet to be a novel adjunct to chemotherapy.

Failure of clinical cancer therapy is attributed mainly to the following two aspects. The first technical difficulty for successful chemotherapy is the site-specific delivery of adequate chemotherapeutics to the tumor site with minimal undesirable systemic toxicities. Recently, biodegradable polymeric nanoparticles composed of amphilic copolymers have attracted intense interest as a promising tumor-targeted drug delivery system. ${ }^{16,17}$ The characteristic structure of amphilic copolymers enables itself to self-assemble into nanoscaled core-shell spherical structures with the hydrophobic part (eg, poly(caprolactone) [PCL]) as the inner core and the hydrophilic part (eg, poly(ethylene glycol) [PEG]) as the outer shell. Therefore, the hydrophobic core is easy to incorporate lipophilic drugs, which exhibits a sustained manner of drug release by slow degradation of the polymer. The outer shell formed by the hydrophilic part (PEG) enables the nanoparticles to escape from the scavenging of the reticuloendothelial systems effectively, thereby leading to a long circulation time in vivo. ${ }^{18}$

Moreover, nanoparticulate drug delivery systems were proven to be preferentially located in the tumor tissue by enhanced permeability and retention effect. ${ }^{19}$ However, systemic administration failed to achieve high drug concentration in the tumor, although it seems that an antitumor effect was augmented in addition to reduced side effects. ${ }^{20}$ Accordingly, local-regional chemotherapy has emerged as an effective method to eradicate tumors. Compared to free drugs, drug-loaded nanoparticles are characterized by the controlled release of incorporated drugs, which may overcome the deficiency of drug retention in the tumor and may reach a satisfying outcome in improving antitumor efficacy when combined with intratumoral delivery. Previous work in the authors' laboratory has demonstrated the enhanced anticancer effect of cisplatin-loaded nanoparticles against liver cancer by intratumoral delivery. ${ }^{16}$

It has been previously reported that Tet-loaded nanoparticles induce more cell apoptosis than free Tet by significantly elevating intracellular reactive oxygen species levels. ${ }^{21}$ Moreover, since the chemoresistance to Ptx correlates with intracellular antioxidant capacity, codelivery of Ptx and Tet generate a synergistic antitumor effect. Basing on the findings above, it has been demonstrated that the coadministration of Ptx and Tet by nanoparticles leads to more intracellular reactive oxygen species induction, which could efficiently enhance the cytotoxicity of Ptx by sequential inhibition of the reactive oxygen species-dependent Akt pathway and activation of apoptotic pathways. ${ }^{15}$

The purpose of the current study was to provide a novel therapeutic strategy that could amplify the antitumor effect of Ptx by employing traditional Chinese medicine as a modulator. This novel approach utilizes polymeric nanoparticles as a drug carrier for the codelivery of Ptx and Tet intratumorally for optimal therapeutic efficacy. Biodegradable core-shell methoxy PEG-PCL nanoparticles incorporating Ptx and Tet simultaneously were prepared, and the in vivo efficacy was examined via intratumoral administration in hepatic carcinoma models.

\section{Material and methods Materials}

Ptx and Tet were kindly provided by Zhejiang Haizheng Pharmaceutical Co, Ltd (Taizhou, China). According to the authors' previous work, PCL20k-PEG4k nanoparticles were used for their higher drug loading content (DLC) and encapsulation efficiency (EE). ${ }^{16}$ 3-(4,5-dimethylthiazol-2-yl)2,5-diphenyltetrazolium bromide (MTT) was purchased from Sigma-Aldrich Corporation (St Louis, MO). All other chemicals were of analytical grade and used without further purification.

Mouse hepatic carcinoma cell line H22 was obtained from Shanghai Institute of Cell Biology (Shanghai, China). The cells were cultured in Roswell Park Memorial Institute 1640 medium with 10\% fetal bovine serum and $100 \mathrm{U} / \mathrm{mL}$ penicillin-streptomycin (Invitrogen/Life Technologies, Carlsbad, CA) at $37^{\circ} \mathrm{C}$ in a water-saturated atmosphere with $5 \%$ carbon dioxide.

\section{Formulation and characterization of nanoparticles}

Ptx/Tet-coloaded nanoparticles were prepared by a nanoprecipitation method as described previously. ${ }^{15}$ Briefly, a predetermined amount of methoxy PEG-PCL block copolymers and Tet and Ptx were dissolved in an aliquot of acetone. The obtained organic solution was added dropwise into ten different volumes of distilled water under gentle stirring at room temperature. The solution was dialyzed in a Spectra POR2 dialysis membrane, (Spectrum 
Lab, Houston, TX) (molecular weight cutoff $12 \mathrm{kDa}$ ) to thoroughly remove the acetone. The resulted bluish aqueous solution was filtered through a $0.22-\mu \mathrm{M}$ filter membrane (GVWP04700; Millipore, Billerica, MA) to remove nonincorporated drugs and copolymer aggregates. Drug-free nanoparticles were produced in a similar way by eliminating drugs. Solutions of drug-loaded nanoparticles and empty nanoparticles were then lyophilized for further utilization. The feeding ratio of nanoparticles to cryoprotectant is 1:5, with Pluronic ${ }^{\circledR}$ F68 (BASF, Ludwigshafen, Germany) as the cryoprotectant.

The mean diameter and size distribution were measured before lyophilization by photon correlation spectroscopy (dynamic light scattering) (BI-9000AT; Brookhaven Instruments Corporation, Holtsville, NY). Zeta potential was measured by laser Doppler anemometry (ZetaPlus; Brookhaven Instruments).

\section{DLC and EE}

The concentration of Ptx and Tet were assayed on an LC-10 AD high-performance liquid chromatography system (Shimadzu Corporation, Tokyo, Japan) equipped with a Shimadzu ultraviolet detector and an Agilent C-18, $5 \mu \mathrm{M}$, $200 \mathrm{~mm} \times 4.6 \mathrm{~mm}$ reversed-phase high-performance liquid chromatography analytical column (Agilent Technologies, Santa Clara, CA). The mobile phase for Tet consisted of methanol (spectral grade; Merck KGaA, Darmstadt, Germany)/double-distilled water/ethylamine (90/10/0.05 volume/volume/volume) pumped at a flow rate of $1.0 \mathrm{~mL} /$ minute, with a determination wavelength of $282 \mathrm{~nm}$. The concentration of Tet was determined based on the peak area at the retention time of 4.83 minutes by reference to a calibration curve.

The mobile phase for detecting Ptx consisted of acetonitrile (spectral grade; Merck)/double-distilled water (58/42 volume/volume) pumped at a flow rate of $1.0 \mathrm{~mL} /$ minute, with a determination wavelength of $228 \mathrm{~nm}$. The concentration of Ptx was determined based on the peak area at the retention time of 7.3 minutes by reference to a calibration curve.

The following equations were applied to calculate the DLC and EE:

$$
\begin{array}{r}
\text { DLC }(\%)=\text { Weight of the drug in nanoparticles } \\
\text { /weight of the nanoparticles } \times 100 \\
\text { EE }(\%)=\text { Weight of the drug in nanoparticles } \\
\text { /weight of the feeding drugs } \times 100
\end{array}
$$

\section{In vitro cytotoxicity studies}

The in vitro drug-induced cytotoxic effects were measured by MTT reduction assay. H22 cells were seeded at a density of 10,000 cells per well and immediately exposed to a series of doses of free Ptx alone, Ptx plus Tet, and $\mathrm{Ptx} /$ Tet nanoparticles, with dimethyl sulfoxide treatment (concentration $<0.1 \%$ ) as a negative control at $37^{\circ} \mathrm{C}$. After treatment, $0.1 \%$ volume of MTT was added to each well, and the plate was further incubated at $37^{\circ} \mathrm{C}$ for another 4 hours. Dimethyl sulfoxide $(200 \mu \mathrm{L})$ was added to each well to solubilize the MTT-formazan product after removal of the medium. Absorbance at $570 \mathrm{~nm}$ was measured with a multiwell spectrophotometer (BioTek, Winooski, VT). Growth inhibition was calculated as a percentage of the untreated controls, which were not exposed to drugs.

\section{In vivo antitumor efficacy}

Imprinting control region mice (Institute of Cancer Research [ICR] mice were purchased from the animal center of Drum Tower Hospital [Nanjing, China]) implanted with murine hepatoma cell line $\mathrm{H} 22$ were used to qualify the relative efficacy of Ptx/Tet nanoparticles delivered intratumorally. The mice were raised under specific pathogen-free circumstances and all of the animal experiments were performed in full compliance with guidelines approved by the Animal Care Committee at Drum Tower Hospital (Nanjing, China). The mice were subcutaneously injected at the left axillary space with $0.1 \mathrm{~mL}$ of cell suspension containing 4-6 × 10 $\mathrm{H} 22$ cells. Treatments were started 7-8 days postimplantation. The mice whose tumor reached a tumor volume of $100 \mathrm{~mm}^{3}$ were selected and this day was designated as day zero.

Animals received a single injection volume of $\sim 0.2 \mathrm{~mL}$ with a 21 -gauge needle placed in the center of the tumor. The intratumoral injections were infused for about 10 seconds, and the needle was allowed to remain in place for an additional 10-15 seconds and removed through another direction. On day zero, the mice were randomly divided into eight groups, with each group being composed of ten mice. The mice were treated with free Ptx, Ptx nanoparticles, Ptx/Tet nanoparticles, empty nanoparticles, and saline, respectively. Ptx was administered at doses of $10 \mathrm{mg} / \mathrm{kg}$. Ptx nanoparticles were administered as a saline solution at the equivalent Ptx doses of $10 \mathrm{mg} / \mathrm{kg}$. Ptx/Tet nanoparticles were administered as a saline solution at the equivalent Ptx doses of 5, 10, and $20 \mathrm{mg} / \mathrm{kg}$, respectively, with the Tet dose set at $15 \mathrm{mg} / \mathrm{kg}$.

All mice were tagged, and the tumors were measured every other day with calipers during the period of study. The 
tumor volume was calculated by the formula $\left(\mathrm{W}^{2} \times \mathrm{L}\right) / 2$, where $\mathrm{W}$ is the tumor measurement at the widest point, and $\mathrm{L}$ is the tumor dimension at the longest point. Relative tumor volume (RTV) was calculated by the formula $\left(\mathrm{V}_{\mathrm{n}} / \mathrm{V}_{0}\right)$, where $\mathrm{V}_{\mathrm{n}}$ is the tumor volume measured at the corresponding day, and $\mathrm{V}_{0}$ is the tumor volume measured at Day 0 . Another antitumor indicator is therapeutic group/control group $\%$, which was calculated by the formula $\left(\mathrm{T}_{\mathrm{RTV}} / \mathrm{C}_{\mathrm{RTV}}\right)$, where $\mathrm{T}_{\mathrm{RTV}}$ is the RTV of the experimental group, and $\mathrm{C}_{\mathrm{RTV}}$ is the RTV of the control group.

Each animal was weighed at the time of treatment so that the dosage could be adjusted to achieve the $\mathrm{mg} / \mathrm{kg}$ amounts reported. Animals also were weighed every other day throughout the experiment. At the end of the experiment, survival curves were calculated by Kaplan-Meier analysis and comparisons were made with the logrank test using SPSS $^{\circledR} 13.0$ software (IBM Corporation, Armonk, NY).

\section{Results}

\section{Particle size and loading efficiency of $\mathrm{Ptx} /$ Tet nanoparticles with different feeding ratios}

As previously reported, Ptx/Tet nanoparticles remain stable for 2 weeks at room temperature. With increasing time, the size of Ptx/Tet nanoparticles showed less variation during the observation period. ${ }^{15}$ The particle sizes and zeta potentials of nanoparticles with different feeding ratios of Ptx and Tet were measured by dynamic light scattering (Table 1). The mean diameter of Ptx/Tet nanoparticles (feeding ratio 1:1) was around $77 \mathrm{~nm}$, which is the smallest among the four kinds of nanoparticles. All four nanoparticle types exhibited a negative zeta potential slightly below $0 \mathrm{mV}$ (Table 1 ).

Figure 1 indicates the drug loading efficiency of Ptx/Tet nanoparticles with different feeding ratios. When the feeding ratio of Ptx and Tet was 1:1, it reached the highest yield with a DLC of $>10 \%$ for the two drugs (Figure 1A). Similarly, the EE of the two drugs with 1:1 feeding ratio was $>80 \%$,

Table I Mean particle size of five kinds of nanoparticles

\begin{tabular}{llll}
\hline $\begin{array}{l}\text { Feeding ratio } \\
\text { Ptx:Tet }\end{array}$ & $\begin{array}{l}\text { Mean particle } \\
\text { size }(\mathbf{n m})^{\mathrm{a}}\end{array}$ & $\begin{array}{l}\text { Zeta potential } \\
(\mathbf{m V})^{\mathbf{a}}\end{array}$ & Polydispersity \\
\hline I:3 & $83.1 \pm 5.7$ & $-6.5 \pm 1.2$ & $0.16 \pm 0.09$ \\
I:2 & $88.3 \pm 8.4$ & $-5.7 \pm 2.1$ & $0.17 \pm 0.08$ \\
I:I & $77.2 \pm 1.9$ & $-6.6 \pm 2.1$ & $0.14 \pm 0.09$ \\
$2: 1$ & $79.6 \pm 4.6$ & $-6.1 \pm 0.7$ & $0.15 \pm 0.08$ \\
$3: 1$ & $90.3 \pm 7.3$ & $-5.3 \pm 0.9$ & $0.17 \pm 0.07$ \\
\hline
\end{tabular}

Note: ${ }^{\text {aThe standard deviation value was obtained from three measurements of a }}$ single batch.

Abbreviations: PTX, paclitaxel; Tet, tetrandrine. which is higher than that of the other three kinds of nanoparticles (Figure 1B). The hydrophobicity of the two drugs and their affinity with the hydrophobic core (PCL) resulted in high loading efficiency. Therefore, the Ptx/Tet nanoparticles formed in the current research provide a satisfactory DLC and EE.

\section{Anticancer efficacy of Ptx/Tet nanoparticles against $\mathrm{H} 22$ cells}

In the in vitro cytotoxicity test, blank nanoparticles were demonstrated to be nearly nontoxic to H22 cells, with inhibition rate $<15 \%$ even at a high concentration of $800 \mu \mathrm{g} / \mathrm{mL}$ (Figure 2A). The concentration of Tet was fixed to $2 \mu \mathrm{M}$, a nontoxic dose which leads to less than 5\% cell death. As shown in Figure 2B, free Ptx, Ptx plus Tet, and Ptx/Tet nanoparticles had a dose-response effect against $\mathrm{H} 22$ cells. Moreover, Ptx plus Tet and Ptx/Tet nanoparticles generated significantly more cell death than free Ptx at a series of equivalent doses. The half maximal inhibitory concentration value of Ptx in cells receiving either Ptx plus Tet (11.22 \pm $1.57 \mathrm{nM})$ or Ptx/Tet nanoparticles $(11.07 \pm 0.97 \mathrm{nM})$ was significantly lower compared to cells receiving free Ptx $(18.50 \pm 1.35 \mathrm{nM})$.

\section{In vivo evaluation of $\mathrm{Ptx} /$ Tet nanoparticles in $\mathrm{H} 22$ xenograft model}

Figure 3A depicts changes of tumor volume by intratumoral administration in $\mathrm{H} 22$ tumor-bearing mice. Ptx/Tet nanoparticles at equivalent Ptx doses of 5, 10, and $20 \mathrm{mg} / \mathrm{kg}$ effectively inhibited tumor growth and displayed a dosedependent antitumor efficacy, while no obvious antitumor effect was observed in the group receiving $10 \mathrm{mg} / \mathrm{kg}$ free Ptx or Ptx nanoparticles. The difference in tumor volume between the group receiving $5 \mathrm{mg} / \mathrm{kg}$ Ptx/Tet nanoparticles and $10 \mathrm{mg} / \mathrm{kg}$ Ptx nanoparticles was significant $(P<0.05)$, as was the difference between the group receiving $10 \mathrm{mg} / \mathrm{kg}$ Ptx nanoparticles and saline $(P<0.05)$. Tumor volumes in groups receiving saline and empty nanoparticles increased rapidly from day five, with their mean volumes reaching more than $5000 \mathrm{~mm}^{3}$ on day 15 . In comparison, groups receiving $10 \mathrm{mg} / \mathrm{kg}$ or $20 \mathrm{mg} / \mathrm{kg}$ Ptx/Tet nanoparticles showed retarded tumor growth, with their mean volumes $<2000 \mathrm{~mm}^{3}$ on day 15. Most importantly, Ptx/Tet nanoparticles exhibited more efficient antitumor efficacy than Ptx nanoparticles by delaying tumor growth. Statistical analysis revealed that the group receiving $10 \mathrm{mg} / \mathrm{kg}$ Ptx/Tet nanoparticles had significantly smaller tumors when compared to the group receiving the corresponding dose of Ptx nanoparticles $(P<0.05)$. 

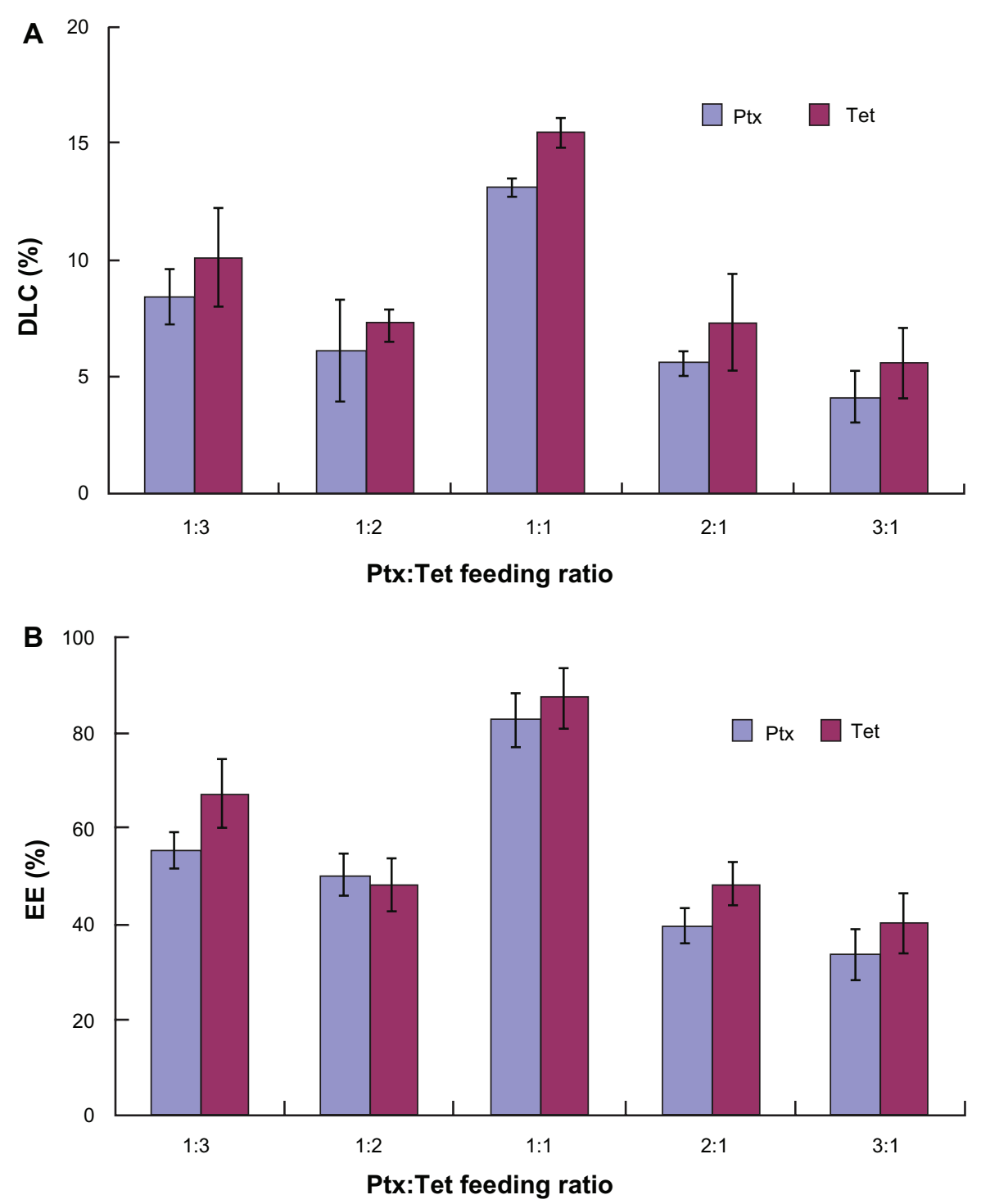

Figure I (A) Drug loading content and (B) encapsulation efficiency of paclitaxel/tetrandrine nanoparticles with different feeding ratios. Abbreviations: DLC, drug loading content; EE, encapsulation efficiency; Ptx, paclitaxel; Tet, tetrandrine.

Figure 3B and C show the change of RTV and therapeutic group/control group $\%$ by intratumoral administration in H22 tumor-bearing mice. The variation of RTV and therapeutic group/control group\% correlated with the trend of tumor volume (Figure 3A), which demonstrated the superior anticancer efficiency of Ptx/Tet nanoparticles against Ptx nanoparticles or free Ptx. An analysis of body weight variations generally defined the adverse effects of the different therapy regiments (Figure 3D). All groups demonstrated favorable results without any obvious body weight loss even at the highest dose. However, it was observed that the mice receiving free Ptx were in a weak state in movement and spirit, whereas no obvious alteration was observed in the nanoparticle-treated animals.
Figure 4 indicates the survival curves of mice receiving different treatments. It was noted that $>60 \%$ of the mice receiving Ptx/Tet nanoparticles survived at the end of the experiment (day 40). However, none of the mice survived to the end in the group receiving saline, empty nanoparticles, and $10 \mathrm{mg} / \mathrm{kg}$ free Ptx. An extremely significant difference was observed between the group receiving free Ptx and the group receiving Ptx nanoparticles $(P<0.01)$ or Ptx/Tet nanoparticles $(P<0.01)$. Moreover, there was a significant difference $(P=0.043)$ between the group receiving the equivalent dose of Ptx/Tet nanoparticles and Ptx nanoparticles (Ptx dose: $10 \mathrm{mg} / \mathrm{kg}$ ). Therefore, in vivo evaluation demonstrated for the first time that coadministration of Ptx and Tet by nanoparticles, when delivered intratumorally, 


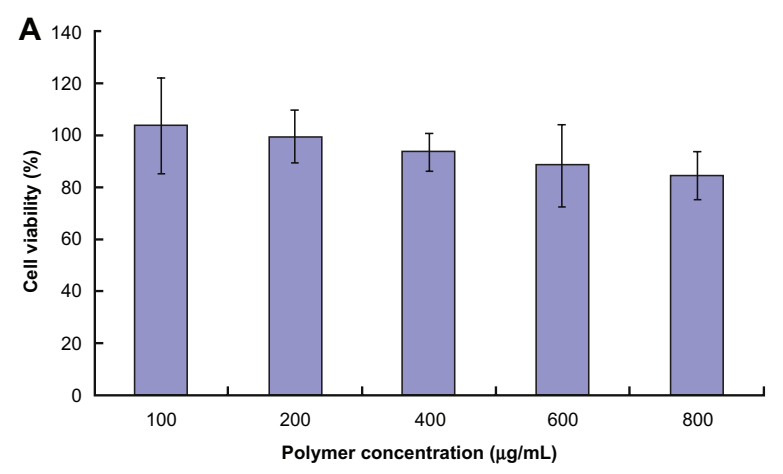

B

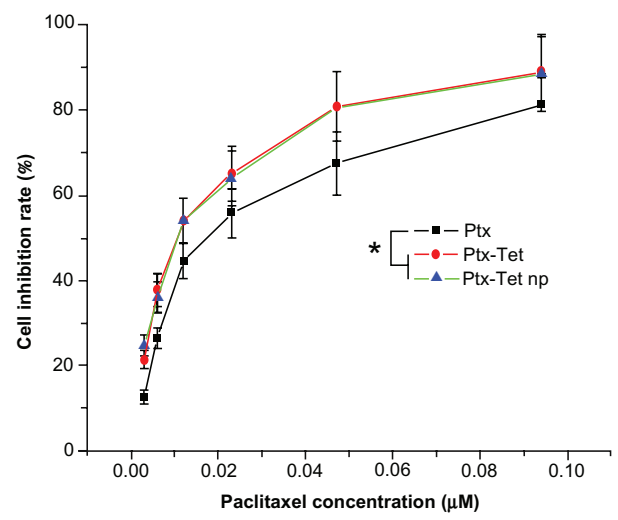

Figure 2 In vitro cytotoxicity of (A) blank nanoparticles and (B) free paclitaxel, paclitaxel plus tetrandrine, and paclitaxel/tetrandrine nanoparticles against $\mathrm{H} 22$ cells for 48 hours.

Note: Data are presented as mean \pm standard deviation $(n=3)$.

Abbreviations: Ptx, free paclitaxel; Ptx-Tet, paclitaxel plus tetrandrine; Ptx-Tet np, paclitaxel/tetrandrine nanoparticles. exhibits significantly increased antitumor efficacy with longer survival time.

\section{Discussion}

Clinically, successful chemotherapy with Ptx needs to reduce the toxic side effects against normal tissues and avoid the detriments caused by intolerable solvents. Different methods were designed to maximize localization of the drug to the tumor while minimizing systemic toxicity. Recently, drug delivery systems have been engineered for the delivery of Ptx. ${ }^{22-24}$ However, in a preliminary study, it was found that Ptx-loaded nanoparticles with amphilic copolymer as the carrier were unstable in the aqueous phase and were easy to aggregate. It was previously reported that Tet could effectively stabilize Ptx-loaded nanoparticles when it was coencapsulated with Ptx. ${ }^{15}$ In the current study, the loading efficiency of Ptx/Tet nanoparticles was detected by varying the feeding ratio of Ptx and Tet. As shown in Figure 1, the feeding ratio greatly influenced the loading efficiency of the codrug-loaded nanoparticles. It was observed that the highest DLC and EE were achieved when the feeding ratio of Ptx and Tet was 1:1. It was also found that more feeding of Tet than Ptx led to a higher loading efficiency.

Moreover, it was demonstrated in the author's laboratory that Tet could synergistically enhance the anticancer effect of Ptx against gastric cancer cells. ${ }^{14,15}$ Therefore, coencapsula-
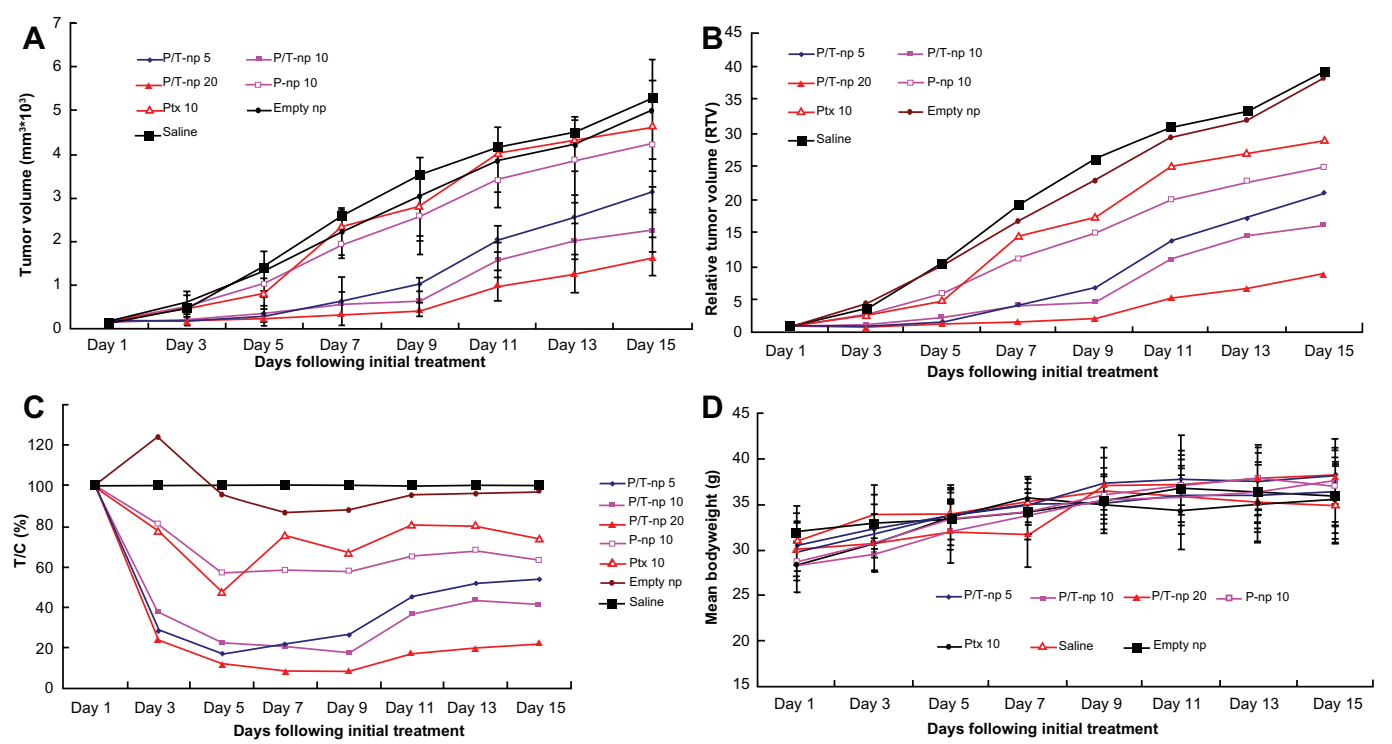

Figure 3 Changes in (A) tumor volume, (B) relative tumor volume, (C) therapeutic group/control group\%, and (D) body weight of H22-transplanted imprinting control region mice receiving different therapy treatments. The mice were treated with different protocols on day zero (arrow), ie, when tumor volume measured $100 \mathrm{~mm}^{3}$. Notes: Data in $(\mathbf{A})$ and $(\mathbf{D})$ are presented as mean \pm standard deviation; $* P<0.05$ versus the group receiving $10 \mathrm{mg} / \mathrm{kg}$ paclitaxel nanoparticles; $* * P<0.05$ versus the group receiving $5 \mathrm{mg} / \mathrm{kg}$ paclitaxel/tetrandrine nanoparticles.

Abbreviations: empty np, empty nanoparticles; P-np 10, paclitaxel nanoparticles in a saline solution at equivalent paclitaxel dose of $10 \mathrm{mg} / \mathrm{kg}$; P/T np, paclitaxel/tetrandrine nanoparticles in a saline solution at equivalent paclitaxel dose of 5,10 , and $20 \mathrm{mg} / \mathrm{kg}$ with tetrandrine dose set at $10 \mathrm{mg} / \mathrm{kg}$; Ptx 10 , free paclitaxel at a dose of $10 \mathrm{mg} / \mathrm{kg}$; saline, vehicle; T/C, therapeutic group/control group. 


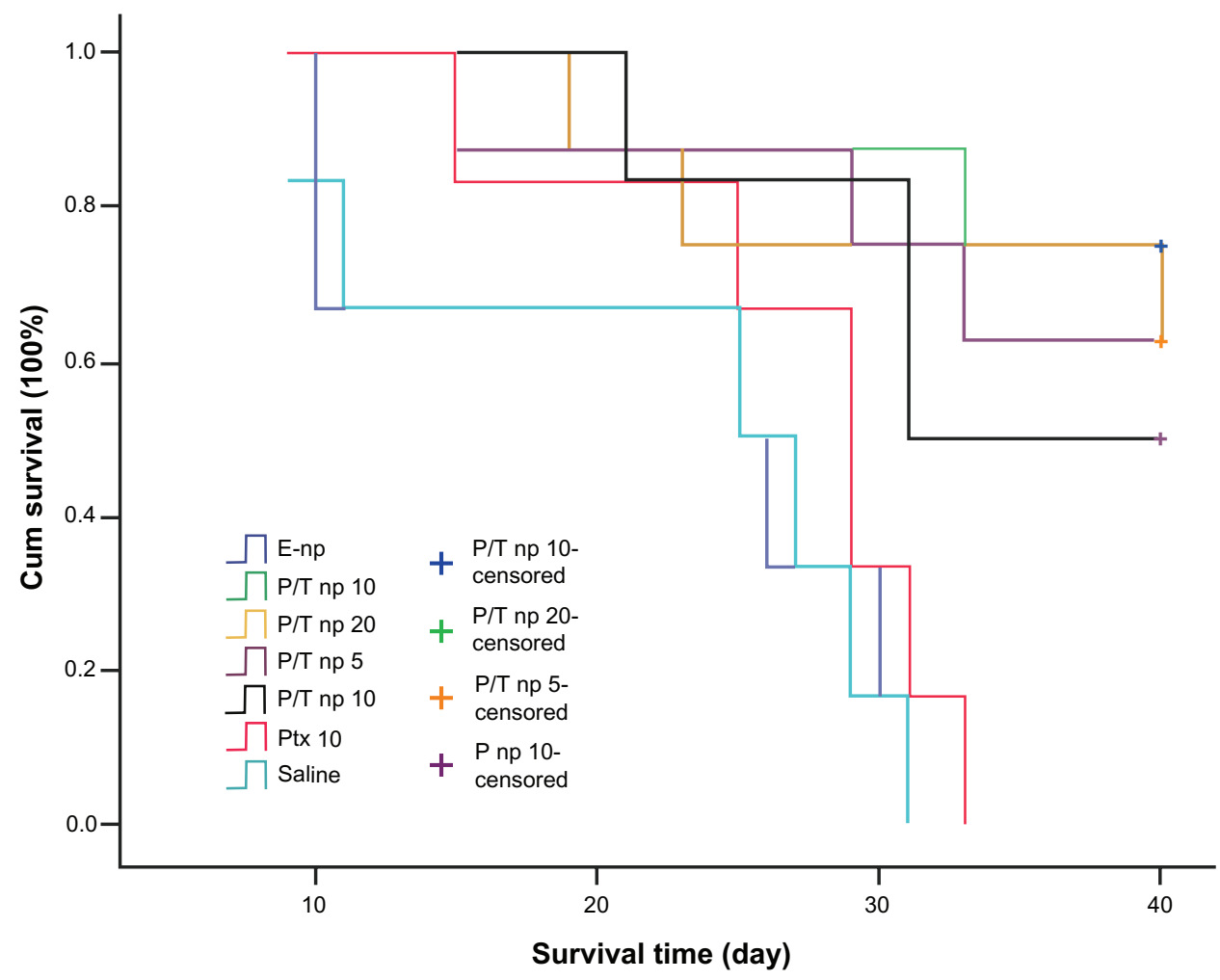

Figure 4 Kaplan-Meier curves showing the survival of tumor-bearing mice treated with different protocols.

Note: The different agents were delivered intratumorally when tumor volume measured $100 \mathrm{~mm}^{3}$.

Abbreviations: cum, cumulative; empty np, empty nanoparticles; P-np I0, paclitaxel nanoparticles in a saline solution at equivalent paclitaxel dose of I0 mg/kg; P/T np, paclitaxel/tetrandrine nanoparticles in a saline solution at equivalent paclitaxel dose of 5,10 , and $20 \mathrm{mg} / \mathrm{kg}$ with tetrandrine dose set at $10 \mathrm{mg} / \mathrm{kg}$; Ptx 10 , free paclitaxel at a dose of $10 \mathrm{mg} / \mathrm{kg}$; saline, vehicle.

tion of Tet and Ptx into nanoparticles not only shows better stability than Ptx-loaded nanoparticles, but also retains the synergistic anticancer efficiency of Tet and Ptx. The in vitro cytotoxicity test indicated that a nearly nontoxic dose of Tet significantly improved the cytotoxicity of Ptx whether or not delivered by nanoparticles. The findings reported in the current and previous studies demonstrate the potential anticancer efficiency of Ptx/Tet nanoparticles in gastrointestinal cancer. ${ }^{15}$

In vivo evaluation in the mice hepatoma model showed the superior antitumor efficacy of Ptx/Tet nanoparticles when delivered by intratumoral injection. To the authors' knowledge, the characteristic pharmacodynamics and pharmacokinetics of intratumoral delivery determines the therapeutic efficacy of Ptx/Tet nanoparticles. As a site-specific delivery to a tumor nodule, the codrug-loaded nanoparticles will accordingly induce a higher local drug concentration than systemic delivery. ${ }^{25}$ Although Ptx/Tet by intratumoral administration cannot achieve as high an initial concentration as free drugs, the sustained release of the encapsulated drugs is capable of delivering its antitumor efficacy constantly. Therefore, it is highly reasonable that released drugs from the intratumorally delivered nanoparticles remain in the interstitial space of the tumor for a longer time compared to normal tissue and exerts a protracted tumor-eliminating effect locally.

Planned modifications of the codrug-loaded nanoparticles utilized in this study are under active consideration as a part of this ongoing research. Further development on the basis of multidrug administration by nanoparticles will be fully reviewed in human orthotopic xenograft models in order to further expand the parameters of this current research. Thus, together with the current findings, it is of great value in future experiments to focus on codrug delivery systems.

\section{Conclusion}

This study reported a stable codrug-loaded nanoparticle formed by amphilic methoxy PEG-PCL block copolymers. Tet and Ptx was coincorporated into the nanoparticles with relatively high loading efficiency. In vitro studies proved the superior cytotoxicity of Ptx/Tet nanoparticles in a dose-dependent manner against H22 cells. In vivo evaluation showed that Ptx/Tet nanoparticles, when delivered intratumorally, exhibited significantly improved antitumor efficacy and, 
moreover, substantially increased the overall survival in an established H22-transplanted mice model.

\section{Acknowledgments}

This work was supported by the National Natural Science Foundation of China (number 81001077 to Xiaolin Li).

\section{Disclosure}

The authors report no conflicts of interest in this work.

\section{References}

1. Jemal A, Siegel R, Ward E, Hao Y, Xu J, Thun MJ. Cancer statistics, 2009. CA Cancer J Clin. 2009;59(4):225-249.

2. American Cancer Society. Cancer Facts and Figures 2012. Atlanta, GA: American Cancer Society; 2012.

3. Diaz JF, Andreu JM. Assembly of purified GDP-tubulin into microtubules induced by taxol and taxotere: reversibility, ligand stoichiometry, and competition. Biochemistry. 1993;32(11):2747-2755.

4. Strumberg D, Erhard J, Harstrick A, et al. Phase I study of a weekly $1 \mathrm{~h}$ infusion of paclitaxel in patients with unresectable hepatocellular carcinoma. Eur J Cancer. 1998;34(8):1290-1292.

5. Chao Y, Chan WK, Birkhofer MJ, et al. Phase II and pharmacokinetic study of paclitaxel therapy for unresectable hepatocellular carcinoma patients. Br J Cancer. 1998;78(1):34-39.

6. Dorr RT. Pharmacology and toxicology of Cremophor EL diluent. Ann Pharmacother. 1994;28(Suppl 5):S11-S14.

7. Bissery MC, Nohynek G, Sanderink GJ, Lavelle F. Docetaxel (Taxotere): a review of preclinical and clinical experience. Part I: preclinical experience. Anticancer Drugs. 1995;6(3):339-355.

8. Kang HS, Kim YH, Lee CS, Lee JJ, Choi I, Pyun KH. Anti-inflammatory effects of Stephania tetrandra S. Moore on interleukin-6 production and experimental inflammatory disease models. Mediators Inflamm. 1996;5(4):280-291.

9. Meng LH, Zhang H, Hayward L, Takemura H, Shao RG, Pommier Y. Tetrandrine induces early G1 arrest in human colon carcinoma cells by down-regulating the activity and inducing the degradation of G1-Sspecific cyclin-dependent kinases and by inducing p53 and p21Cip1. Cancer Res. 2004;64(24):9086-9092.

10. Wu JM, Chen Y, Chen JC, Lin TY, Tseng SH. Tetrandrine induces apoptosis and growth suppression of colon cancer cells in mice. Cancer Lett. 2010;287(2):187-195.

11. Oh SH, Lee BH. Induction of apoptosis in human hepatoblastoma cells by tetrandrine via caspase-dependent Bid cleavage and cytochrome c release. Biochem Pharmacol. 2003;66(5):725-731.

12. Fu LW, Zhang YM, Liang YJ, Yang XP, Pan QC. The multidrug resistance of tumour cells was reversed by tetrandrine in vitro and in xenografts derived from human breast adenocarcinoma MCF-7/adr cells. Eur $J$ Cancer. 2002;38(3):418-426.
13. Choi SU, Park SH, Kim KH, et al. The bisbenzylisoquinoline alkaloids, tetrandine and fangchinoline, enhance the cytotoxicity of multidrug resistance-related drugs via modulation of P-glycoprotein. Anticancer Drugs. 1998;9(3):255-261.

14. Wei J, Liu B, Wang L, Qian X, Ding Y, Yu L. Synergistic interaction between tetrandrine and chemotherapeutic agents and influence of tetrandrine on chemotherapeutic agent-associated genes in human gastric cancer cell lines. Cancer Chemother Pharmacol. 2007;60(5): 703-711.

15. Li X, Lu X, Xu H, et al. Paclitaxel/tetrandrine coloaded nanoparticles effectively promote the apoptosis of gastric cancer cells based on "oxidation therapy." Mol Pharm. 2012;9(2):222-229.

16. Li X, Li R, Qian X, et al. Superior antitumor efficiency of cisplatinloaded nanoparticles by intratumoral delivery with decreased tumor metabolism rate. Eur J Pharm Biopharm. 2008;70(3):726-734.

17. Shao J, Zheng D, Jiang $Z$, et al. Curcumin delivery by methoxy polyethylene glycol-poly(caprolactone) nanoparticles inhibits the growth of C6 glioma cells. Acta Biochim Biophys Sin (Shanghai). 2011;43(4):267-274.

18. Herold DA, Keil K, Bruns DE. Oxidation of polyethylene glycol by alcohol dehydrogenase. Biochem Pharmacol. 1989;38(1):73-76.

19. Maeda $H$. The enhanced permeability and retention (EPR) effect in tumor vasculature: the key role of tumor-selective macromolecular drug targeting. Adv Enzyme Regul. 2001;41(2):189-207.

20. Idani H, Matsuoka J, Yasuda T, Kobayashi K, Tanaka N. Intra-tumoral injection of doxorubicin (Adriamycin) encapsulated in liposome inhibits tumor growth, prolongs survival time and is not associated with local or systemic side effects. Int J Cancer. 2000;88(4):645-651.

21. Li X, Zhen D, Lu X, et al. Enhanced cytotoxicity and activation of ROSdependent c-Jun NH2-terminal kinase and caspase-3 by low doses of tetrandrine-loaded nanoparticles in Lovo cells - a possible Trojan strategy against cancer. Eur J Pharm Biopharm. 2010;75(3):334-340.

22. Ma X, Wang H, Jin S, Wu Y, Liang XJ. Construction of paclitaxel-loaded poly (2-hydroxyethyl methacrylate)-g-poly (lactide)-1,2-dipalmitoylsn-glycero-3-phosphoethanolamine copolymer nanoparticle delivery system and evaluation of its anticancer activity. Int J Nanomedicine. 2012; 7:1313-1328.

23. Wang J, Mongayt D, Torchilin VP. Polymeric micelles for delivery of poorly soluble drugs: preparation and anticancer activity in vitro of paclitaxel incorporated into mixed micelles based on poly(ethylene glycol)-lipid conjugate and positively charged lipids. J Drug Target. 2005;13(1):73-80.

24. Shenoy D, Little S, Langer R, Amiji M. Poly(ethylene oxide)-modified poly $(\beta$-amino ester) nanoparticles as a $\mathrm{pH}$-sensitive system for tumor-targeted delivery of hydrophobic drugs. 1 . In vitro evaluations. Mol Pharm. 2005;2(5):357-366.

25. Walter KA, Tamargo RJ, Olivi A, Burger PC, Brem H. Intratumoral chemotherapy. Neurosurgery. 1995;37(6):1128-1145.
International Journal of Nanomedicine

\section{Publish your work in this journal}

The International Journal of Nanomedicine is an international, peerreviewed journal focusing on the application of nanotechnology in diagnostics, therapeutics, and drug delivery systems throughout the biomedical field. This journal is indexed on PubMed Central, MedLine, CAS, SciSearch ${ }^{\circledR}$, Current Contents ${ }^{\circledR} /$ Clinical Medicine,

\section{Dovepress}

Journal Citation Reports/Science Edition, EMBase, Scopus and the Elsevier Bibliographic databases. The manuscript management system is completely online and includes a very quick and fair peer-review system, which is all easy to use. Visit http://www.dovepress.com/ testimonials.php to read real quotes from published authors. 